\title{
Assessment of Healthcare Utilization among Women Experiencing Childbirth in Suburban Nepal
}

\author{
Shelley Xu, B.A.1,2 \\ Cyril Blavo D.O., MPH \& TM ${ }^{1,2}$ Sujan Babu Marahatta Ph.D. ${ }^{2,3}$ Mayur Banjara, MPH ${ }^{2,4}$ \\ Rakchya Amatya ${ }^{3}$ Patrick Hardigan, Ph.D, MBA ${ }^{1,2}$ Nadia Anderson, B.S., MPH $^{5}$
}

\begin{abstract}
The postnatal period (birth to 6 weeks of age) is a high-risk period for mothers and their newborns. Despite significant improvements in the Nepalese healthcare system over the last decade, there is still a high incidence of maternal and neonatal deaths during the postnatal period. These factors, in addition to existing socioeconomic barriers, discourage many women from returning to these facilities for postnatal care. This study therefore, aims to determine the extent of healthcare utilization among women with recent childbirth experience in the Mahalaxmi municipality in the Lalitpur district of Nepal. This study is a descriptive cross-sectional needs assessment utilizing a pre-tested survey instrument administered to ninety-eight women who were randomly selected from the semi-urban Mahalaxmi municipality. All the respondents reported that they received ante-natal care during their pregnancy. A majority of the women reported that they received perinatal care from physicians. The results of the study showed that although there was a significantly high utilization of ante-natal and perinatal care among women with recent childbirth experience, most of the women were unaware of the availability or necessity of postnatal care, despite the accessibility of physicians and healthcare facilities.
\end{abstract}

Keywords: Childbirth, maternal health, postnatal care, pregnancy, utilization

\section{Introduction}

Nepal has had a tumultuous socio-political history over the past two decades that has only begun to settle down within the last few years. This instability has greatly impacted accessibility to healthcare, such that Nepal currently ranks 149 out of 189 on the Human Development Index (United Nations Development Programme, 2018). In Nepal there is lower life expectancy rates, higher prevalence of transmissible disease, and lack of reproductive care due to poor healthcare infrastructure. Major changes have been made by the government in the last few years, including implementation of the Safe Motherhood Plan and Newborn Long

\footnotetext{
${ }^{1}$ Corresponding Author: Shelley Xu, B.A., Address: $4905^{\text {th }}$ Ave, Apt 4519, Fort Lauderdale, FL 3330 I USA Email: sx8@mynsu.nova.edu INova Southeastern University, Dr. Kiran C. Patel College of Osteopathic Medicine,

2International Health Initiatives, Inc.

3Manmohan Memorial Institute of Health Sciences, Nepal

${ }^{4} \mathrm{New}$ lersey Department of Health

${ }^{5}$ Florida International University
} 
Term Plan, which offer financial incentives to utilize maternal healthcare services and increase training of professional midwives (Kearns, 2017). Nevertheless, more progress is yet to be made.

The postnatal period (birth to 6 weeks of age) is a high-risk period for mothers and their newborns; a period of time when most maternal and infant deaths in Nepal related to childbirth is known to occur. The World Health Organization (WHO) recommends at least three postnatal care visits after birth (on day 3, between days 7-14, and at 6 weeks) (WHO, 20/3). In general, most neonatal deaths due to asphyxia occur within the first week of life. Over $60 \%$ of cases of sepsis and pathological jaundice, both common causes of neonatal deaths, develop during the first week of life (WHO, 2013). During postnatal care visits, both maternal and newborn health is assessed. Infants are evaluated for health conditions including the presence of poor feeding behaviors, abnormal body temperatures, atypical body habitus, irregular movements, heart irregularities and respiratory difficulties. Mothers are evaluated for presence of hemorrhage, urinary incontinence, abnormal bowel function, perineal hygiene, and postpartum depression (World Health Organization, 2013). Studies have shown that increased utilization of postnatal care can reduce maternal and infant morbidity and mortality, as well as maternal postpartum depression (Lobato, 20I4). However, as of 2015 , only $58 \%$ of mothers in Nepal have utilized postnatal care services (WHO, 2013).

Many factors may contribute to the poor utilization of postnatal care by Nepalese women, including national policies and priorities, cultural practices, autonomy issues, proximity to healthcare facilities, availability of skill birthing assistants, and quality of health care facilities (Kearns, 2017). Economic needs resulting in poor infrastructure has made it challenging for the nation to provide quality skilled birthing attendants as well as emergency obstetrics and newborn care in rural and semi-urban areas of Nepal (Lobato, 20I4). In addition, many rural Nepalese women are heavily influenced by family members to not seek antenatal or postnatal care (Simkhada, 2010). There is a perceived need to conduct research into postnatal utilization in Nepal in order to create sustainable interventions in reproductive health and birthing practices in rural and semi-urban communities (Khanal, 2014).

The Mahalaxmi municipality, where this study is conducted, lies in Lalitpur district of Nepal. Lalitpur is a relatively populated district of 468,132 (compared with the 1,744,240 of Kathmandu, the capital of Nepal) (Government of Nepal, 2013). At 385 sq. km.it is the third smallest of the 14 regions of $\mathrm{Nepal}$ and is a very densely populated region. The major religions of the population in this district are Buddhism and Hinduism, with a smaller but sizeable Christian population. Currently, the Lalitpur district is ranked $2^{\text {nd }}$ out of all the Nepalese districts by the Human Development Index, just after Kathmandu (Morgan, 20I4).

\section{Methods}

This study is a descriptive cross-sectional needs study assessment. All 98 of the survey respondents were women from the semi-urban Mahalaxmi municipality in Lalitpur district of Nepal. Residents are defined as living in Lalitpur for at least 6 months. Only females ranging 
between ages 16 and 39 and who had children were interviewed for this study. Each woman was asked a list of questions from a structured questionnaire regarding demographic information (religion, caste, marital status, number of children), utilization of antenatal, perinatal and postnatal care, awareness of postnatal care, accessibility of healthcare facilities, and female autonomy.

The study was conducted in Mahalaxmi municipality of Lalitpur district, one of the three districts of Kathmandu valley, situated in the northern part of Central Development Region. The municipality consists of 19 Wards. There are altogether 5 Health posts (HP) located in Wards I, 7, 10, 13, and 16. There is also a teaching hospital in Ward 16.

A simple random sampling technique was used for the study. As per the District Public Health Office of Lalitpur district, the estimated target population of mothers of children under two years of age of Mahalaxmi municipality in the year 2015 were 2,749. The random sample of 98 was taken from the sampling frame. Participants were recruited to the study in person. Investigators knocked on doors without any coercion in order to invite individuals to participate.

Inclusion criteria for selection included: I) Mothers who have at least one child under two years of age; 2) Residents of the municipality living there for at least 6 months. The exclusion criteria included mothers who were severely ill and/or could not speak. The tool for data collection was a structured questionnaire. The technique used for data collection was interviewing.

\section{Results}

\section{Social History}

All the 98 respondents were married. Sixty-eight (68\%) of them were from the Janajati caste and $18 \%$ were from the Brahmin Chettri caste. The age range of respondents at the birth of their last child prior to the study was grouped as follows: I6-20 y.o. (I2\%); 2 I- 25 y.o. (29\%); $26-29$ y.o. (34\%); 30-35 y.o. (20\%); 36-39 y.o. (5\%).

It was self-reported that $13 \%$ of the respondents were illiterate. The highest level of educational attainment was at the primary level for 2 respondents. The distribution of secondary education attainment level among the respondents was: lower secondary (9\%); secondary (29\%); higher secondary (22\%). Fourteen (I4\%) of the respondents were found to achieve a higher education level.

Seventy-five percent (75\%) of the respondents were unemployed. Of the 22 employed, $9 \%$ were in business, $7 \%$ worked for non-governmental organizations, $2 \%$ were farmers and $2 \%$ relied on daily wages.

The husbands of all 98 respondents (100\%) were employed. The distribution of employment among the husbands was: farming (2\%); government service (6\%); non-governmental organizations (12\%); business (34\%). Twenty percent (20\%) of the husbands depended on daily wages, and $25 \%$ of the husbands were working abroad. The highest level of educational 
attainment among the husbands was: primary (7\%); lower secondary (9\%); secondary (32\%); higher secondary (19). Nineteen (19) of them were in higher education and 4 were illiterate.

\section{Household Decision-making}

Eighty-one percent $(81 \%)$ of the mothers surveyed reported that their last pregnancy was planned. Approximately $20 \%$ reported that their last pregnancy was not planned. Relative to household decision-making, the respondents reported that these decisions were made primarily by the husband ( $42 \%)$; both husband and wife (28\%); by the family (23\%) and least often by the wife (8\%) (See Figure I).

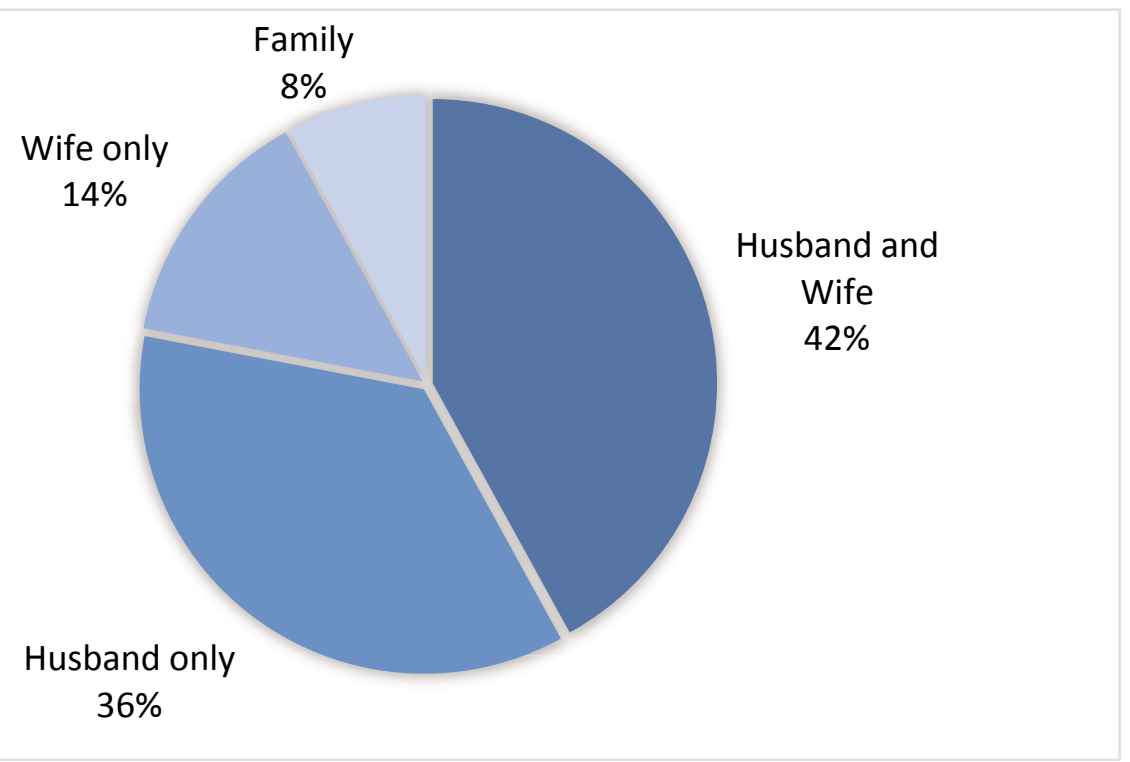

Figure I. Household decision making

Relative to the children's education, the respondents reported that these decisions were made primarily by both the husband and wife ( $42 \%)$; by the husband (36\%); the wife (14\%); and least often by the family (8\%). On the decision to consult a healthcare provider, the respondents reported that such a decision is made primarily by both the husband and wife (39\%); by the wife (34\%); by the husband (23\%); and least often by the family (5\%) (See Figure 2 ). 


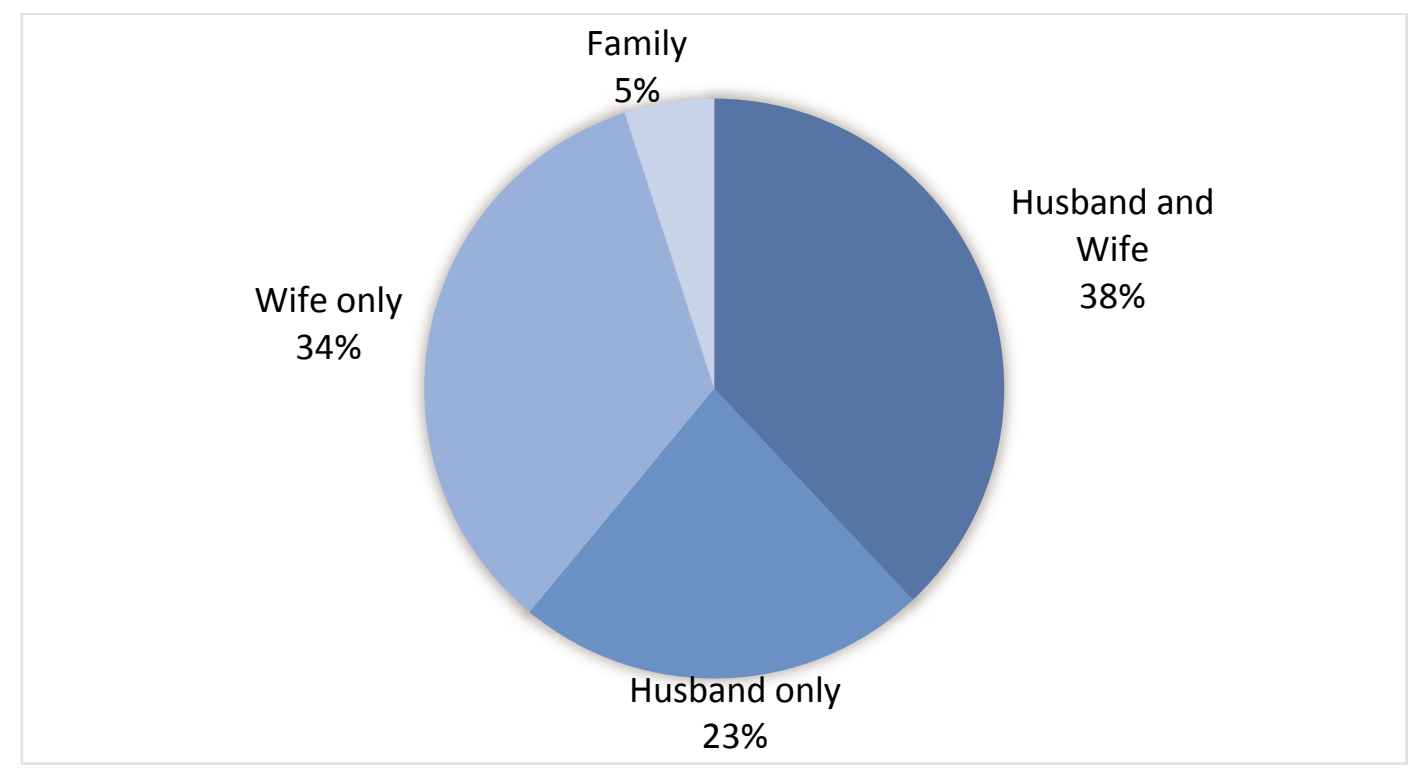

Figure 2. Decision to consult healthcare provider

\section{Antenatal Care Utilization}

In the survey of antenatal care (pre-natal care) utilization, all the 98 respondents reported that they visited an antenatal care center (ANC) (See Figure 3). Eighty-nine percent (89\%) of the mothers reported that they visited an ANC over 4 times. Seven percent (7\%) reported 3 visits; $2 \%$ reported 2 visits and the rest (2\%) did not receive any antenatal care. Eighty-nine percent $(89 \%)$ reported that their caregiver at the antenatal care visit was a doctor. Approximately 10\% reported that they received antenatal care from a nurse, midwife or auxiliary. Ninety-four percent $(94 \%)$ of the respondents reported that at the time of their antenatal care visit they were taking iron supplementation. Eighty-eight percent (88\%) of the mothers surveyed denied that they had experienced any danger signs in their pregnancy at the time of the antenatal visit, and $98 \%$ of the mothers reported satisfaction with their antenatal care.

\section{Birthing Facility Utilization}

Ninety-three percent $(93 \%)$ of the respondents reported that their latest birthing experience occurred in a healthcare facility. Seven percent (7\%) reported having their latest birthing experience at home. Among the facilities in which the birthing occurred, $82 \%$ reported that it occurred at a government healthcare facility, while $11 \%$ reported that it occurred at a private healthcare facility. Among the mothers surveyed, $91 \%$ reported their delivery was facilitated by a doctor. Fewer than 10\% reported that their delivery was facilitated by a nurse, auxiliary, midwife, Female Community Health Volunteer (FCHV) or traditional birth attendant. Seventynine percent $(79 \%)$ of the mothers reported that they did not experience any danger signs during their delivery. Twenty-two percent (22\%) reported that they experienced some danger 
signs during their pregnancy. Nevertheless, $94 \%$ of the mothers reported satisfaction with their birthing experience.

\section{Perinatal Care}

Eighty-six per cent $(86 \%)$ of the mothers reported that they received a checkup from their doctor within 24 hours after delivery. Fourteen percent (14\%) reported that they did not receive postnatal care within 24 hours post-partum (See Figure 3).

\section{Postnatal Care Utilization}

Eighty-three percent (83\%) reported that they did not receive any additional postnatal visit within 6 weeks post-partum (See Figure 3). When asked whether they were familiar with postnatal care, $65 \%$ of the respondents (mothers) denied awareness of postnatal care. Approximately $35 \%$ of the mothers reported that they have heard about postnatal care (See Figure 3). Among the media through which respondents gained awareness of postnatal care are: health workers, newspaper, friends, radio or television, and family.

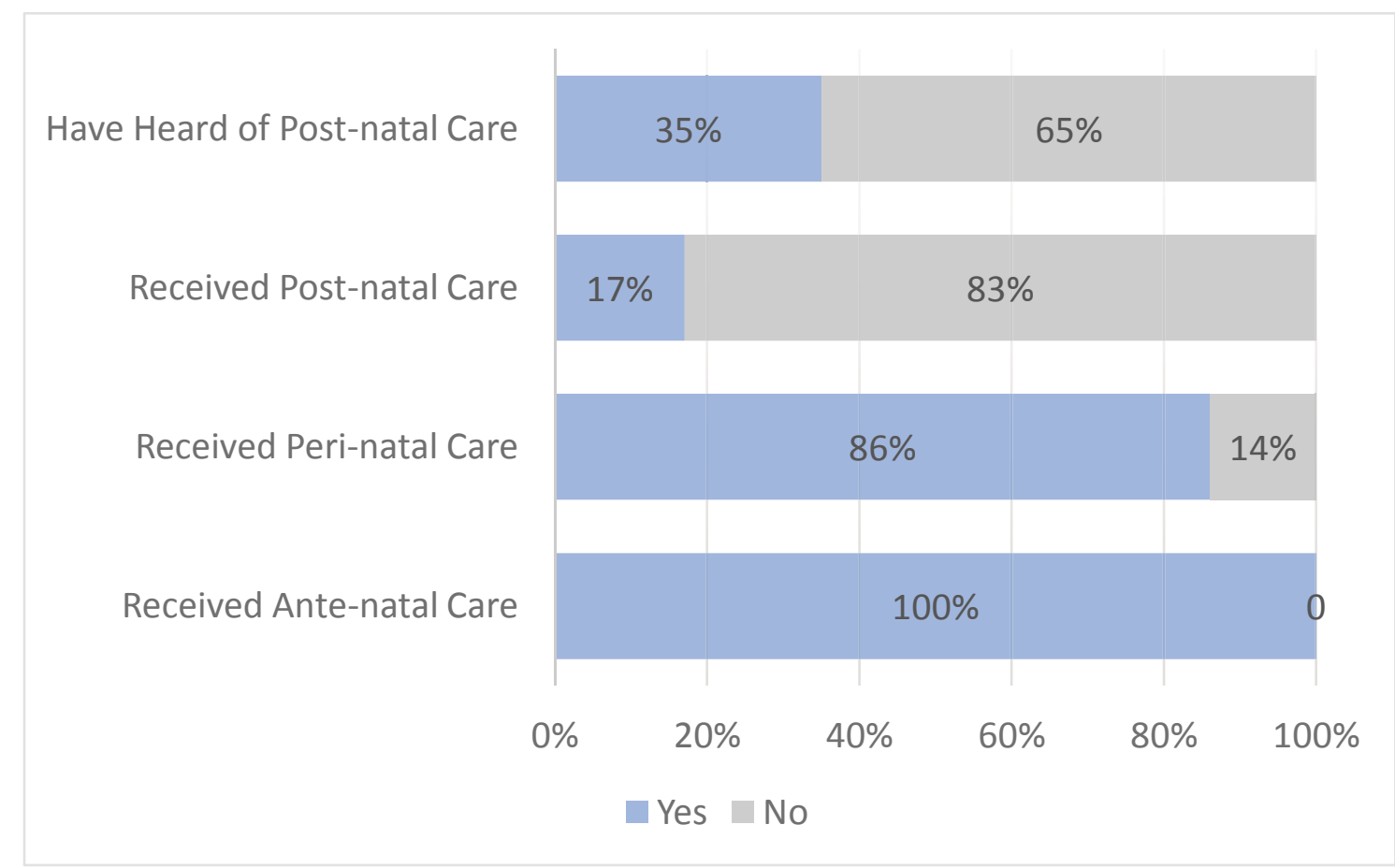

Figure 3. Survey of women of childbearing age: utilization of ante-, peri- and post-natal care

\section{Availability of Maternal Care Resources}

With respect to accessibility of healthcare facilities to the mothers surveyed, $87 \%$ reported that the nearest healthcare facility was within 30 minutes of travel. Twenty percent $(20 \%)$ of the respondents reported that the nearest facility was over 30 minutes away. All the respondents (100\%) reported that postnatal care services were available at their healthcare facility. Fifty-two percent (52\%) of the mothers interviewed received some type of perinatal or 
postnatal care. At the postnatal care visit, only $33 \%$ reported that they were examined. Eighteen percent $(18 \%)$ reported that they received family planning services at the postnatal care visit. Sixteen percent $(16 \%)$ reported that they received information on nutrition. None of the 62 respondents (mothers) who had received postnatal care reported receiving counseling, iron supplementation, breastfeeding education, vitamin A supplementation or immunization at the time of the postnatal care visit. Forty-seven percent $(47 \%)$ of the mothers reported that they were given a referral or transportation for an emergency at the postnatal visit.

Regarding the types of postnatal care services they received, the respondents identified the following: immunization (9l\%); vitamin supplementation (78\%); medical examination (72\%); iron supplementation (53\%); family planning (14\%); counseling (8\%). None of the respondents identified breastfeeding education or nutrition education among the postnatal services they received after delivery.

\section{Discussion}

These survey results showed differences in antenatal, perinatal, and postnatal care utilization among the respondents. In the survey of antenatal care utilization, all 98 respondents reported that they visited an antenatal care center, with $89 \%$ having visited an antenatal care center over 4 times. The majority of women (86\%) from the Lalitpur district received perinatal care from physicians 24 hours immediately after birth.

However, relative to postnatal care (up to 6 weeks after birth), there was a significant reduction in utilization. Eighty-three percent $(83 \%)$ of the women reported that they did not receive any additional postnatal visit within 6 weeks post-partum, and $65 \%$ of the women reported that they were unaware of postnatal care. Healthcare facilities were found to be accessible in the Mahalaxmi municipality, according to $87 \%$ of the women, who reported that the nearest healthcare facility was within $\mathbf{3 0}$ minutes of travel. Physicians were also found to be accessible, with $91 \%$ of women reporting that their births were facilitated by a physician.

Compared to our study where $17 \%$ of women received postnatal care within 2 weeks of birth, and $89 \%$ of women received perinatal care within 24 hours after birth, similar studies showed varying rates of postnatal and perinatal care utilization in Nepal. A study done in the Kaski district of $\mathrm{Nepal}$ in $201 \mathrm{I}$ revealed $0 \%$ of the women received postnatal care within 24 hours of birth (perinatal care) and 32.6\% received postnatal care within I week of birth (Karkee, 20I6). The Kaski district ranks 5 th out of the 75 districts in Nepal in the Human Development Index, while the Lalitpur district in our study ranks $2^{\text {nd }}$ (Karkee, 2016). Comparatively, the national data from the Nepal Demographic and Health Survey (NDHS) in 2016 reported that $57 \%$ of women in the overall population received postnatal care within 2 days after birth. Of the total number of women who received postnatal care, $64 \%$ were urban women and $48 \%$ were rural women (Ministry of Health, 2016).

Based on the results of these studies, our team has concluded that when surveying mothers, it is important for the definition of postnatal care to be explicitly differentiated from perinatal 
care, which is defined as 24 hours after birth. The differences in reported postnatal care utilization among studies may be due to women misunderstanding the two definitions, as seen by the differences in categorical data portrayed by these two studies. Further differences may be attributed to varying degrees of social, economic, and environmental nuances among each district. These factors play a role in the poor utilization of postnatal care by Nepalese women, including the political will of the government, cultural practices, female autonomy, proximity to healthcare facilities, availability of skill birthing assistants, and quality of health care facilities.

\section{Political Climate and Infrastructure}

Nepal is listed as one of the many fragile and conflicted-area situation (FCS) countries in the United Nations. Even as a post-conflict nation, it still struggles with fragility due to political instability, and therefore maternal and neonatal health greatly suffer. The length of fragility in the country correlates with higher maternal mortality ratios per live childbirth, and these FCS countries are found to document the highest rates of maternal and neonatal morbidity and mortality in Asia and the Middle East (Goplan, 2017). Much of the country remains rural, and $76 \%$ of the population is dependent on agriculture for its income (Kearns, 2017). In some of the most remote parts of the country, there are no working roads, so it can take up to two days to reach the nearest hospital. In these areas, women are required to find porters because cars cannot or will not pick them up (Morgan, 20I4). In many villages, the major modes of transportation are still by donkey or mule (Khatri, 2017).

\section{Autonomy of Women and Influence of Family Members}

Nepal currently ranks II8/160 in the gender inequality index (United Nations Development Programme, 2018). Women have lower literacy and education rates than men and work mostly as unskilled laborers. Although the majority of women are employed in agriculture, $61 \%$ of married women are not paid for their work (Kearns, 2017). A 2016 study done in the Kapilvastu district, a rural district in Western Nepal, showed that women's autonomy was low, especially in terms of financial autonomy (Bhandari, 2018). Women's autonomy in Nepal is closely correlated with many factors such as age, education, occupation and income. However, the most important predicting factors of autonomy were the woman's education level, their husbands' education levels, and their household socioeconomic status (Bhandari, 2018). The better educated the women and their husbands are, the more likely it is that they will use maternal healthcare services.

A 2014 study conducted in Kathmandu, the urban and dense capital of Nepal, surveyed mothers, their husbands, and their mothers-in-law to rank the perceived decision-maker of the family when it came to antenatal healthcare services. Antenatal care utilization was found repeatedly to be a good predictor for postnatal care utilization (Simkhada, 2010). Results showed that pregnant young adult women and teens were more likely to defer to their husbands' decisions on utilization of maternal healthcare, whereas most adult women felt they, themselves, were the most influential participants in antenatal care and delivery care decisionmaking. Overall, the husband was found to play the most influential role in maternal healthcare 
decision-making during pregnancy of women in all age groups (Upadhyay, 2014). These results, however, may be solely limited urban environments similar to that of Kathmandu.

In the rural areas of Nepal, tradition more heavily favors the influence of mothers-in-law on their daughters-in-law (Upadhyay, 20I4). Usually, women marry young, and after marriage, the wife traditionally lives with the husband's family. Oftentimes, young girls become pregnant and most drop out of school, perpetuating the poorly educated, young motherhood cycle. Nepalese men are not conventionally involved in childbirth due to the traditional belief in some areas that God becomes angry if they are to touch women in labor or post-labor (Khatri, 2017). Therefore, the mother-in-law becomes the decision-maker of maternal health issues. Many rural Nepalese women are not allowed to go for antenatal or postnatal checkups unless allowed by their mother-in-law (Simkhada, 2010).

Interviews of women, their husbands, and their mothers-in-law were conducted in both rural and urban districts of Nepal and showed mostly negative effects the mothers-in-law had on antenatal and postnatal care. Many mothers-in-law, themselves, did not receive maternal health care during their pregnancies, and therefore did not believe it was necessary for their daughters-in-law to do so (Simkhada, 2010). Instead, they saw household chores as a priority for their daughters-in-law, and may push them to work throughout their pregnancy, up until their delivery. In some cases, where the daughters-in-law had access to earning or were able to obtain funds elsewhere, they have managed to find a way to obtain care (Simkhada, 20I0).

\section{Health Care Facilities and Skilled Birthing Attendants}

NDHS reported that $81 \%$ of women who delivered in a health facility received a postnatal check within 2 days after the delivery (Ministry of Health, 2016). Giving birth at a health facility is a positive predictor of receiving postnatal care (Simkhada, 2010). Therefore, it is important to look at the causes for poor antenatal care and reasons for homebirths. In Nepal, there are about 1500 birthing centers available in rural areas, but only $10 \%$ of births take place in those facilities (Khatri, 2017). Birthing centers in Nepal are the lowest tier of health facility where women may give birth. These birthing centers are standardized across the country, and consist of 2 patient rooms, a treatment room, and a storage area (Morgan, 2014). These centers must consist of one Auxiliary Nurse Midwife (ANM) who is trained as a Skilled Birthing Assistant (SBA), but often are staffed by ANMs who are not additionally trained (Khatri, 2017). The SBA accreditation consists of a 2 month long training program in addition to the 18 month long ANM training. In the SBA program, they specifically learn emergency obstetric and neonatal care, including use of antibiotics, anticonvulsants, and resuscitation (Khatri, 20I7). Many newly trained SBAs testified that they could assist deliveries with much more confidence after the focused training program, and performed fewer unnecessary episiotomies (Morgan, 20I4).

In addition to the many reasons for home birth mentioned above, there seems to be an overall distrust of the SBAs within some Nepalese communities due to miscommunication and previous experiences. There is also the perception that it is only necessary to attend a birthing center if one encounters complications during birth, so women often do not feel the need to 
seek trained birthing help (Morgan, 2014). Even though birthing facilities are supposed to be open around the clock, oftentimes the SBA was not present when women arrived at the birthing center for antenatal care, childbirth, or postnatal care (Khatri, 2017). As a result, professionally trained midwives would have to take over, but often lived too far to arrive at the facilities in a timely fashion.

SBAs are also expected to monitor women for up to 8 hours after delivery and observe any postpartum complications. However, many of them note that there was not enough space in the facilities to keep women after delivery for postnatal care. Mothers would often leave the birthing facility just 2 hours after giving birth (Morgan, 2014). In addition, birthing facilities, unlike hospitals, do not usually have electricity. This makes it difficult to keep the mother and baby warm, especially during the winter (Morgan, 20l4). In a study conducted in Western Nepal, both patients and SBAs alike felt that, overall, the most important factor was for each birthing facility to have at least 2 SBAs on call to deliver quality care and manage lifethreatening complications (Morgan, 2014). With improvement of birthing facilities and increased SBA staffing, women might be more willing to seek facility births and be compliant with postnatal care.

\section{Conclusion}

This study reveals that although there is a high utilization of antenatal and perinatal care in Lalitpur district of Nepal, most women do not utilize postnatal care. These findings of poor utilization of postnatal care occur in spite of the accessibility of physicians and healthcare facilities in Lalitpur. Our results indicate that there is a need to educate women about the necessity of postnatal care in order to further reduce maternal morbidity and mortality in Nepal. It is also necessary to increase the accessibility of birthing centers in more rural areas of Nepal, and ensure they are appropriately staffed. The ultimate objective is to empower and encourage women to give birth in these facilities and return for postnatal care in order to reduce maternal morbidity and mortality. Facility births and use of antenatal care are good predictors of postnatal care utilization, which makes antenatal care compliance an important starting point for an intervention strategy in rural and semi-urban areas of Nepal. Programs to educate women about the necessity of professional care during birth and after birth should be instituted to improve the relationship between healthcare providers and mothers. More studies about postnatal care utilization in other municipalities in Nepal should be conducted to better understand utilization across the nation.

\section{Declarations}

Formal approval has been granted from Institutional Review Committee (IRC) of MMIHS and IRB of Nova Southeastern University. The participants were informed and counseled about aims, methods, and anticipated benefits and risks of the study program. Written and verbal informed consents were taken with each and every respondent. No pressure or inducement of any kind will be applied to encourage an individual to a subject of research. During the study 
period all the ethical consideration as well as confidentiality will be maintained to respect human dignity and principle of justice.

\section{References}

Bhandari, T. R., Kutty, V. R., \& Ravindran, T. K. (2016). Women's Autonomy and Its Correlates in Western Nepal: A Demographic Study. Plos One,II(I). doi:I0.137I/journal. pone.0147473

Gopalan, S. S., Das, A., \& Howard, N. (2017). Maternal and Neonatal Service Usage and Determinants in Fragile and Conflict-affected Situations: A Systematic Review of Asia and the Middle-East. BMC Womens Health, I (1). doi: I0.I 186/s I 2905-017-0379-x

Government of Nepal. (2013). Statistical Year Book of Nepal 2013. Kathmandu, Nepal: Central Bureau of Statistics.

Karkee, R., \& Khanal, V. (2016). Postnatal and Neonatal Care after Home Birth: A Communitybased Study in Nepal. Women and Birth,29(3). doi:I0.1016/j.wombi.20I5.10.003

Kearns, A., Onda, S., Caglia, J., Tuncalp, O., Langer, A. (2017). Postnatal Care in Nepal: Components of Care, Implementation Challenges, and Success Factors. Retrieved from https://cdn2.sph.harvard.edu/wp-content/uploads/sites/32/2014/09/HSPH-Nepal6.pdf

Khanal, V., Adhikari, M., Karkee, R., \& Gavidia, T. (2014). Factors Associated with the Utilisation of Postnatal Care Services among the Mothers of Nepal: Analysis of Nepal Demographic and Health Survey 20II. BMC Womens Health, I4(I). doi:10.1186/14726874-14-19

Khatri, R. B., Dangi, T. P., Gautam, R., Shrestha, K. N., \& Homer, C. S. (2017). Barriers to Utilization of Childbirth Services of a Rural Birthing Center in Nepal: A Qualitative Study. Plos One, I2(5). doi:10.137I/journal.pone.0177602

Lobato, G., Brunner, M. A., Dias, M. A., Moraes, C. L., \& Reichenheim, M. E. (2012). Higher Rates of Postpartum Depression among Women Lacking Care after Childbirth: Clinical and Epidemiological Importance of Missed Postnatal Visits. Archives of Womens' Mental Health, I5(2), I45-I46. doi:I0. I007/s00737-0I2-0256-4

Ministry of Health, Nepal; New ERA; and ICF. 2017. Nepal Demographic and Health Survey 2016. Kathmandu, Nepal: Ministry of Health, Nepal.

Morgan, A., Soto, E. J., Bhandari, G., \& Kermode, M. (20l4). Provider Perspectives on the Enabling Environment Required for Skilled Birth Attendance: A Qualitative Study in Western Nepal. Tropical Medicine \& International Health,19(12), I457-I465. doi: 10.11 II/tmi. 12390

Falle, T. Y., Mullany, L. C., Thatte, N., Khatry, S. K., Leclerq, S. C., Darmstadt, G. L., Katz, J. and Tielsch, J. M. (2009). Potential Role of Traditional Birth Attendants in Neonatal Healthcare in Rural Southern Nepal. Journal of Health, Population and Nutrition,27(I). doi:10.3329/jhpn. v27il.3317 
Neupane, S., \& Doku, D. (20/3). Utilization of Postnatal Care Among Nepalese Women. Maternal and Child Health Journal,I7(10), 1922-1930. doi:I0.1007/sI0995-012|218-1

Simkhada, B., Porter, M. A., \& Teijlingen, E. R. (2010). The Role of Mothers-in-law in Antenatal Care Decision-making in Nepal: A Qualitative Study. BMC Pregnancy and Childbirth, IO(I). doi:10.1 I86/147|-2393-10-34

United Nations Development Programme. (2018). Human development reports. Kathmandu,Nepal: United Nations Development Programme.

Upadhyay, P., Liabsuetrakul, T., Shrestha, A. B., \& Pradhan, N. (20/4). Influence of Family Members on Utilization of Maternal Health Care Services among Teen and Adult Pregnant Women in Kathmandu, Nepal: A Cross Sectional Study. Reproductive Health, I I (I). doi: I0.I I86/I742-4755-II-92

World Health Organization. (20I3). WHO recommendations on postnatal care of the mother and newborn. Geneva, Switzerland: World Health Organization. 